Meta

Journal des traducteurs

Translators' Journal

\title{
Traduction de textes de vulgarisation et de textes didactiques : approche pédagogique
}

\section{Monique C. Cormier}

Volume 35, numéro 4, décembre 1990

URI : https://id.erudit.org/iderudit/002393ar

DOI : https://doi.org/10.7202/002393ar

Aller au sommaire du numéro

Éditeur(s)

Les Presses de l'Université de Montréal

ISSN

0026-0452 (imprimé)

1492-1421 (numérique)

Découvrir la revue

Citer cet article

Cormier, M. C. (1990). Traduction de textes de vulgarisation et de textes

didactiques : approche pédagogique. Meta, 35(4), 676-688.

https://doi.org/10.7202/002393ar d'utilisation que vous pouvez consulter en ligne. 


\section{TRADUCTION DE TEXTES DE VULGARISATION ET DE TEXTES DIDACTIQUES : APPROCHE PÉDAGOGIQUE}

MONIQUE C. CORMIER

Université de Montréal, Montréal, Canada

Dans un article précédent (Cormier, 1990), nous avons insisté sur les trois types de difficultés que la traduction technique posait généralement aux étudiants, à savoir la compréhension du texte et du sujet traité, la recherche documentaire et terminologique qui s'y rapporte et la reformulation. Nous avons aussi insisté sur l'avantage d'utiliser une typologie des textes à traduire qui repose sur le public visé. Dans les pages qui suivent, en nous conformant à cette typologie et en nous servant de textes traduits par des étudiants, nous tentons de cerner les écueils particuliers qui guettent les apprentis traducteurs.

\section{TEXTE DE VULGARISATION}

Nous préconisons l'utilisation de textes de vulgarisation en début d'apprentissage de la traduction technique pour plus d'une raison. D'abord, un principe élémentaire en pédagogie veut que l'on commence l'apprentissage par ce qui est relativement facile du point de vue de l'apprenant pour passer ensuite à des éléments plus ardus. Pour ce faire, une progression de la facilité et de la difficulté est tout indiquée. Nous avons déjà défini ce que nous entendions par «facilité» et «difficulté» en traduction technique et insistê sur le fait qu'il faut bien distinguer entre la facilité de compréhension et la facilité de traduction ou de réexpression d'un texte (Ibid., 1990). Par ailleurs, comme la traduction d'un texte de vulgarisation exige généralement peu de recherche documentaire et terminologique, mais qu'en revanche elle nécessite un effort de reformulation particulier, l'introduction du texte de vulgarisation en début d'apprentissage permet d'insister et de travailler sur le «maniement du langage», pour reprendre l'expression de Jean Delisle (1980), c'est-à-dire sur la reformulation.

On peut se demander pourquoi la traduction d'un texte de vulgarisation exige un effort particulier de reformulation. La reformulation d'un texte est le résultat, d'une part, d'équivalences ponctuelles et, d'autre part, de correspondances établies. La traduction d'un texte de vulgarisation fait normalement appel, dans l'ensemble, à davantage d'équivalences inédites que la traduction d'un texte destiné aux spécialistes, lequel comporte davantage de correspondances établies. Sur le plan linguistique, on peut dire que, dans le texte de vulgarisation, le sens est généralement loin de la signification, telle qu'on la trouve dans les dictionnaires, sauf pour des cas ponctuels, notamment les termes. On sait en réalité que le contexte et la situation ont un effet sur le sens et que la connotation, fréquente dans la langue générale, n'est pas sans modifier la charge sémantique que l'on accorde aux mots.

Par contre, dans le texte destiné aux spécialistes, on trouve à peu près l'inverse. Ainsi, le sens a tendance à se rapprocher de la signification, à lui être équivalent. De son côté, la dénotation l'emporte sur la connotation. 
Ces observations sont importantes en traduction. En effet, elles expliquent le recours plus fréquent au transcodage lexical dans la traduction des textes destinés à des spécialistes que dans la traduction des textes de vulgarisation. Comme le sens des mots du texte destiné à des spécialistes correspond souvent à leur signification en langue, la traduction peut alors se faire en grande partie par application de correspondants établis plutôt que par création d'équivalences ponctuelles.

En somme, la traduction d'un texte de vulgarisation exige un effort de reformulation supérieur à celui d'un texte destiné à des spécialistes parce que plus le sens s'éloigne de la signification, plus la traduction doit être le résultat heureux de choix délibérés et éclairés, alors que dans le texte destiné à des spécialistes, pour un grand nombre de termes, sens et signification se recoupent.

Afin d'illustrer ce qui vient d'être dit et de préciser les écueils particuliers de la traduction technique, nous utiliserons un extrait de texte de vulgarisation traduit par des étudiants. On verra que l'analyse des erreurs est nécessaire pour l'établissement de stratégies d'apprentissage.

\section{ASBESTOS: THE PROBLEM GROWS 1}

A new study indicates private buildings may also be at risk.

\section{A Novel, Maybe Deadly, Use}

Meanwhile, scientists funded by the Department of Health and Human Services discovered up to 200,000 California homes that contain air-distribution ducts made of corrugated asbestos paper. Irving Selikoff, of Mount Sinai Medical Center, says he was surprised to find this novel use of asbestos. He and colleague William Nicholson are investigating whether air forced through the ducts carries asbestos fibers. No one knows if similar ducts were used elsewhere - or in what other ways the deadly substance may be infiltrating homes and workplaces.

Asbestos is the collective name for several naturally occurring fibrous minerals used in products as varied as insulation, floor tiles and brake linings. It is heat resistant and strong.

The form of asbestos commonly used in this country is chrysotile, or white asbestos.

The minerals are safe when contained. But if the fibers become loose and airborne, they may be inhaled. If they then become embedded in the lungs, they can cause asbestosis, a scarring of the lungs that is sometimes fatal ; mesothelioma, a rare cancer of the chest or abdomen; lung cancer, especially in smokers; and other cancers.

Ce texte a été distribué à des étudiants à qui l'on a demandé de préciser quelles leur semblaient être les difficultés de traduction. A priori, c'est-à-dire après avoir pris connaissance du texte, mais avant de l'avoir traduit, les seules difficultés qu'ils y ont relevées ont été les termes spécialisés, notamment air distribution ducts, corrugated asbestos paper, fibrous minerals, floortiles, brake linings, chrysotile et mesothelioma, c'est-à-dire des termes que les étudiants, pour la plupart d'entre eux, n'avaient jamais rencontrés².

Quant à nous, nous savions pertinemment que les termes, lorsqu'ils sont inconnus, constituent certes une difficulté, mais une difficulté relativement mineure. Mineure bien sûr dans la mesure où l'étudiant est initié aux principes de la recherche terminologique et sait effectuer une recherche documentaire, mais difficulté tout de même comme on le verra dans les deux exemples qui suivent. 
La traduction des termes énumérés ci-dessus n'a posé aucun problème, sauf dans le cas de l'unité air distribution ducts que plusieurs étudiants ont rendue par «conduites de distribution d'air» alors qu'il s'agissait ici de «conduits d'aération» ou de «conduits de ventilation». Cette erreur n'est en soi pas grave puisqu'elle n'entrave pas la compréhension du message et que le lecteur saisit tout à fait ce dont il est question. Cependant, il faut garder à l'esprit la responsabilité du traducteur en matière de diffusion terminologique. L'écrit impose en effet une langue beaucoup plus spécialisée et précise que l'oral qui utilise une langue plus courante. Contrairement à l'interprète qui dispose d'une plus grande marge de mancuvre terminologique, comme tous ceux d'ailleurs qui s'expriment oralement, le traducteur est tenu de se conformer à une terminologie reconnue. Une erreur comme celle que nous venons de citer peut fournir à l'enseignant l'occasion de rappeler l'importance de la précision terminologique. Par ailleurs, elle peut aussi être l'occasion de montrer la nécessité de procéder, dans certains cas, à une étude synonymique. Ainsi, une étude synonymique des termes «conduit», «conduite» et «canalisation» révèle que si «conduite» et «canalisation» sont souvent interchangeables, «conduit» et «conduite» ne le sont pas.

De même, le terme asbestos a été rendu dans certains cas par «amiante» et dans d'autres, par «asbeste». Interrogés sur la motivation de leur choix, les étudiants ont affirmé que les deux termes figuraient au dictionnaire. Compte tenu du fait que la maladie professionnelle contractée à la suite d'une exposition aux fibres d'amiante porte le nom d'amiantose au Québec et celui d'asbestose en France, il est légitime de se demander si les notions d'amiante et d'asbeste sont équivalentes, c'est-à-dire si les termes sont synonymes et interchangeables. Bien que la consultation de spécialistes ne soit pas à écarter dans de telles situations, l'étude synonymique permet de faire le point avec précision à condition que le traducteur dispose, bien sûr, de suffisamment de temps.

L'étude synonymique effectuée par les étudiants a mis en lumière certaines distinctions. Bien qu'ayant nous-même fait l'étude synonymique auparavant, nous nous sommes abstenue, pour des raisons pédagogiques, d'en faire connaître les résultats avant que les étudiants aient eux-mêmes fait leurs recherches. Certes on ne peut s'attendre que les étudiants effectuent toutes les études synonymiques qu'il serait souhaitable de faire, en raison du temps qu'il faudrait leur consacrer. D'ailleurs, il arrive souvent que les termes en question aient déjà fait l'objet d'une étude synonymique dans des publications. Mais dans le cas où il n'existe aucune étude sur la question, il est bon que les étudiants soient eux-mêmes confrontés à ce type de problème et qu'ils apprennent à le résoudre.

Quant aux distinctions mises en lumière par l'étude synonymique d'amiante et d'asbeste, les documents consultés montrent que ces deux termes sont synonymes et interchangeables quand ils se présentent dans des textes de vulgarisation. Ainsi, les encyclopédies et les dictionnaires généraux les considèrent comme formant une seule et même notion. Par contre, une étude plus poussée dans des ouvrages spécialisés révèle que l'amiante et l'asbeste sont tous deux des silicates fibreux dont la structure est semblable, mais dont la composition chimique diffère (Cormier et Ménassa, 1987). De plus, le terme «amiante» semble être un générique désignant l'ensemble des silicates fibreux de deux groupes de minéraux, c'est-à-dire la serpentine (amiante) et les amphiboles (asbeste). Les termes «amiante» et «asbeste» ne sont donc pas synonymes pour les spécialistes. On peut schématiser leur relation de la façon suivante: 


\section{Langue générale \\ amiante $=$ asbeste}

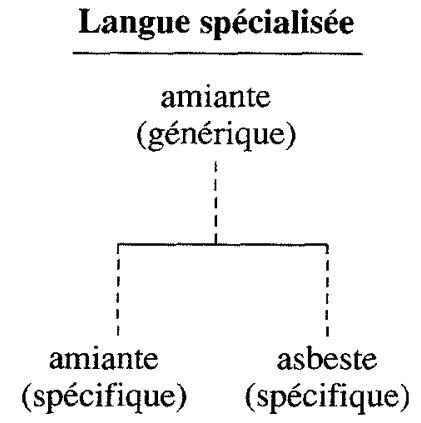

Ces constatations ont des incidences sur la traduction. En effet, si l'on peut au choix utiliser «amiante» ou «asbeste» pour traduire asbestos dans les textes généraux ou de vulgarisation, il en va différemment quand il s'agit de textes destinés à des spécialistes. Il faut alors savoir si l'auteur fait référence à l'amiante ou à l'asbeste, ou encore s'il emploie ce mot de façon générale pour désigner la famille des silicates fibreux. En pareil cas, seules la consultation de spécialistes ou l'étude synonymique permettent de dégager des distinctions aussi précises.

La correction des traductions a en outre confirmé ce que nous pensions, à savoir que les vraies difficultés sont plus subtiles que celles que pose la terminologie. Il faut souligner ici un type d'erreur qui est beaucoup plus grave dans la mesure où la traduction en est opacifiée. Ce type d'erreur est généralement le résultat d'un transcodage inconsidéré résultant de la non-compréhension du message par le traducteur. C'est une erreur grave parce que, comme le dit Marianne Lederer (1981: 176),

[...] le transcodage qui découle d'une non compréhension [sic] du message par le traducteur ne permet pas à l'interlocuteur final une compréhension immédiate.

Une idée mal comprise ne peut être réexprimée clairement dans une autre langue sans que cette «demi-compréhension» ne s'accompagne d'une perte d'information ou d'une grave entrave à la compréhension chez le destinataire.

Voici un exemple de traduction opaque. Par cette expression, il faut entendre une traduction qui, sans être totalement fausse, ne permet pas au lecteur de comprendre le sens à la première lecture et sans avoir recours à l'original. Dans le texte sur l'amiante que les étudiants ont traduit, un passage se lit comme suit:

The minerals are safe when contained. But if the fibers become loose and airborne, they may be inhaled.

Aux fins de la présente étude, nous reproduisons quelques interprétations de la première phrase parmi les plus représentatives de l'échantillonnage.

Les minéraux ne présentent aucun danger lorsqu'ils sont isolés.

Les substances minérales ne présentent aucun danger lorsqu'elles sont contenues.

L'amiante ne présente aucun danger tant qu'il n'entre pas en contact avec l'air.

Les minéraux ne comportent pas de risque s'ils sont contenus.

Les minéraux sont inoffensifs lorsqu'ils sont massifs.

Ces minéraux sont sans danger s'ils sont renfermés. 
Ces minéraux ne présentent pas de danger quand leur stabilité est maintenue.

Hermétiquement scellés, ces minéraux sont inoffensifs.

Par ailleurs, ces minéraux ne posent aucun danger à condition qu'on les garde dans des récipients appropriés.

Ces interprétations sont ici reproduites pour montrer que sans une compréhension juste du sens véhiculé par le message anglais et les connaissances thématiques appropriées, il est très difficile, voire impossible, d'arriver à une réexpression intelligible.

Comme on peut le constater, c'est la traduction du passage qui entoure la notion de contained qui n'a pas été réussie. Si les étudiants sont arrivés à une formulation grammaticalement correcte, leur traduction demeure dans l'ensemble ambiguë et, dans certains cas, totalement fausse. On peut s'interroger sur les raisons qui ont conduit à cette mauvaise traduction.

Il semble à première vue qu'un problème de compréhension se soit posé. Il faut presque avoir vu ou même avoir eu entre les mains un échantillon d'amiante ou, à défaut, avoir eu recours à une illustration, pour bien se le représenter et avoir une image nette des fibres qui le composent. Dès lors, on comprend que l'amiante est fait de fibres et qu'il ne présente aucun danger tant que ses fibres ne se détachent pas. En saisissant bien cette idée, on voit mieux ce que le passage anglais signifie. Ce ne sont donc pas les minéraux ou l'amiante qui doivent être contained, mais bien leurs fibres. Ce que le texte anglais présente implicitement, le français exige qu'on le rende explicitement. On comprend alors mieux que l'amiante est inoffensif tant que ses fibres ne se détachent pas. Ce passage aurait donc pu se traduire, entre autres façons, comme suit :

L'amiante ne présente aucun danger tant que ses fibres ne sont pas libérées.

Si aucun étudiant n'a réussi à proposer une solution satisfaisante, c'est que tous ont sauté une étape importante du raisonnement. Au cours d'une discussion avec eux portant sur l'analyse des erreurs, il est clairement ressorti que la majorité d'entre eux n'avait pas associé le mot contained aux fibres: cette association étant indispensable à la compréhension, il était alors presque impossible d'arriver à une traduction intelligible. La traduction était donc opaque.

Le problème évoqué ci-dessus est bien sûr ponctuel. Considéré isolément, on pourrait même dire qu'il présente peu d'intérêt dans la mesure où les possibilités de trouver à nouveau dans un texte à traduire l'association des termes minerals et contained sont très faibles. En dépit de son caractère ponctuel, cet exemple illustre bien le problème de la non-compréhension et ses conséquences éventuelles sur la traduction. Si, dès le début de l'apprentissage de la traduction technique, l'enseignant n'insiste pas suffisamment sur la nécessité de comprendre avant de rendre, ses efforts pédagogiques risquent d'être vains. L'enseignement de la traduction technique au premier niveau doit viser davantage à faire acquérir aux apprenants une rigueur et une souplesse d'esprit, des méthodes de recherche efficaces, etc., qu'une formulation ou une réexpression de tout premier ordre. Ces étapes sont en effet préalables à la réussite de toute traduction technique.

Â la non-compréhension s'ajoute un autre problème, celui de la terminologie. Le terme désigne toute unité lexicale propre à la langue de spécialité et utilisée exclusivement par des spécialistes ou encore toute unité lexicale empruntée à la langue courante mais qui, employée dans un contexte spécialisé, subit une extension sémantique et acquiert un ou plusieurs sens nouveaux.

Le problème posé par les termes en traduction ne se limite pas à la question des correspondants. Comme le dit Danica Seleskovitch (1975: 43), 
[...] il ne suffit pas de connaître le mot et son équivalent dans l'autre langue; il faut encore connaître la notion ou l'objet qu'il recouvre.

En fait, trouver le correspondant d'un terme peut se comparer à résoudre une équation à quatre variables. Ainsi, pour un terme anglais donné, on dira qu'il peut exister quatre types d'inconnues.

Tout d'abord, le traducteur peut se trouver devant un terme anglais dont il ne connaît ni le signe, ni la notion. Prenons comme exemple le terme anglais clevis qui appartient au domaine des isolateurs de lignes aériennes de transport et de distribution d'électricité et qui a comme correspondant français le terme «chape».

Ensuite, le traducteur peut connaître un signe, mais ignorer la notion qu'il recouvre. Dans cette catégorie peuvent entrer les termes utilisés exclusivement dans la langue de spécialité, comme clevis, mais aussi les mots de la langue courante qui prennent un sens spécialisé dans les textes spécialisés, comme head, shoulder, etc. Selon, par exemple, que le terme head appartient à des domaines aussi variés que l'anatomie, l'astronomie, l'informatique, la construction, l'électronique, etc., la notion est différente. Comme on le disait précédemment, ce ne sont pas nécessairement les termes inconnus qui posent le plus de difficultés aux traducteurs. Danica Seleskovitch (1975: 48) l'a déjà constaté et écrit à ce sujet :

On a souvent tendance à penser que le mot inconnu recèle plus de difficultés, qu'il recouvre des notions plus techniques que le mot connu. Cette réaction est immotivée; la rareté d'un mot ne permet pas plus de conclure à la difficulté de la notion qu'il désigne, que la connaissance de la forme ne permet de conclure à la connaissance du contenu, et donc à la facilité du sujet.

Enfin, le traducteur peut connaître une notion, mais être incapable d'y apposer une étiquette, un signe. Ainsi, lorsque le texte fait référence aux isolateurs dans le domaine des lignes aériennes de transport d'électricité, il peut savoir que certaines pièces ont une extrémité en $U$ dans laquelle un tenon peut se loger et qui est percée de deux trous dans lesquels passe un axe d'assemblage, sans savoir que cette partie porte le nom anglais de clevis.

Enfin, ces inconnues déterminées, il reste au traducteur à trouver un correspondant pour résoudre l'équation de départ. Dans un premier temps, la consultation de dictionnaires bilingues permet de trouver les correspondants potentiels. Dans un deuxième temps, il faut s'assurer que le signifié du terme anglais correspond bien à celui du terme français. Cette vérification faite, on peut dire que clevis est égal à «chape».

Si l'on revient au texte sur l'amiante, on constate que l'unité lexicale contained est fréquemment utilisée dans la langue courante et, en traduction, on lui attribue intuitivement le correspondant «contenu». C'est ce que nous appellerons le «sens premier» de cette unité lexicale. Au moment de traduire une unité comme celle-ci, c'est souvent l'acception la plus courante qui vient d'abord à l'esprit et on oublie parfois l'acception spécialisée qui peut s'y attacher. C'est ce qui s'est passé dans le cas des étudiants qui ont rendu contained par «contenus». L'unité lexicale connue que l'on utilise dans la langue courante, et à laquelle s'attache une couche sémantique supplémentaire dans la langue de spécialité, constitue souvent un piège contre lequel il faut systématiquement mettre en garde les étudiants, tout comme on le fait notamment pour les anglicismes en début d'apprentissage. Qu'un étudiant trouve dans un texte par exemple les termes head ou shoulder, il les traduira presque d'office par «tête» ou «épaule» alors que, selon les domaines d'utilisation, ils pourront avoir comme correspondants «chapiteau», «cuvette», «haut», «pomme», «mouton», etc. et «épaulement», «embase», «collet», «talon», «accotement», etc. 
Nous avons bien sûr relevé d'autres erreurs dans la correction du texte sur l'amiante, erreurs soit de langue, soit de reformulation. Par exemple, la phrase suivante:

He and colleague William Nicholson are investigating ${ }^{3}$ whether air forced through the ducts carries asbestos fibers.

Quelques étudiants n'y ont vu que le sens premier du verbe to investigate et ont traduit par «mènent présentement une enquête», à la place de «cherchent à déterminer si $[\ldots]$ », sans recourir à une exégèse lexicale. Autre exemple, lorsqu'il est question de l'amiante et que le texte anglais dit:

It is heat resistant and strong.

On dira davantage pour traduire l'idée de strong que l'amiante est résistant ou solide plutôt que ferme, robuste ou fort. On utilise en effet les adjectifs «résistant» ou «solide» lorsqu'on veut montrer qu'une chose résiste bien à l'usure, à des forces ou à des actions qui la menacent. On aurait alors pu traduire la phrase de la façon suivante :

Ces minéraux sont incombustibles et résistants.

ou encore

Ces minéraux sont ignifuges et résistants.

Ces deux erreurs relèvent davantage de l'apprentissage de la traduction générale que de celui de la traduction technique. Toutefois l'enseignant ne doit pas les négliger sous prétexte qu'elles ne sont plus de son ressort.

À la lumière de ce qui précède, nous pouvons maintenant faire quelques observations. Notre expérience et l'analyse du texte sur l'amiante nous enseignent que les problèmes de terminologie qui surgissent dans les textes de vulgarisation sont relativement simples. Comme il s'agit généralement d'unités terminologiques non complexes, c'està-dire comportant rarement plus d'un terme, l'utilisation judicieuse des dictionnaires permet la plupart du temps de trouver leur(s) correspondant(s). Par ailleurs, sans que le recours à l'étude synonymique soit nécessaire dans tous les cas, il est bon de faire prendre conscience aux étudiants que les termes utilisés par le grand public d'une part, et par les spécialistes d'autre part, peuvent être différents et qu'il faut savoir les choisir en fonction des destinataires.

En dehors de la question terminologique, une chose apparait tout à fait claire. L'effort de reformulation sera vain tant qu'il sera paralysé ou annihilé par une noncompréhension du texte. C'est l'un des aspects les plus importants dont la didactique de la traduction technique doit tenir compte. Il ne faut pas oublier non plus que le problème de compréhension est un problème nouveau pour l'étudiant qui se présente à un cours de traduction technique de premier niveau. Bien sûr, des problèmes de compréhension de textes se sont déjà posés dans des cours moins avancés, mais l'expérience prouve que l'incompréhension d'un texte général est directement liée à la méconnaissance de la langue de ce texte. L'incompréhension ou la mauvaise compréhension d'un texte technique sont, quant à elles, plutôt liées au contenu spécialisé du message. Il faut donc distinguer l'incompréhension résultant de la mauvaise connaissance d'une langue, qui, de toute façon, ne permet pas d'atteindre le message ni de le rendre dans une autre langue, de l'incompréhension résultant de la mauvaise connaissance du contenu spécialisé et thématique d'un message. S'il est vrai que la connaissance de la langue d'un texte spécialisé à traduire constitue le tout premier obstacle à surmonter, il ne suffit pas de vaincre cet obstacle pour bien rendre le texte, encore faut-il connaître le contenu spécialisé du message à transmettre. Comme le dit Edmond Cary (1985: 28-29) : 
Sans doute pour jouer du violon, faut-il commencer par avoir un violon en main, pour écrire un roman, faut-il avoir du papier et une plume ou une machine à écrire et faut-il savoir former des mots et des phrases. Mais l'essentiel de la musique, l'essentiel de la littérature est ailleurs - et c'est cela qui importe. Pour traduire, que ce soit des poètes ou des brevets techniques, il faut connaître peu ou prou deux langues au moins: mais ce n'est là qu'un point de départ, une des données initiales [...].

\section{TEXTE DIDACTIQUE}

De par sa nature qui vise à instruire, le texte didactique constitue un pont idéal entre le texte de vulgarisation et le texte destiné aux spécialistes.

Avant d'analyser les résultats d'un texte traduit par des étudiants, nous préciserons la marche que nous avons jugé utile de suivre et qui contribue à illustrer notre point de vue.

Lorsqu'on aborde pour la première fois un sujet technique, une question d'ordre pédagogique se pose: comment initier les étudiants au sujet traité ? Comment franchir cette étape préalable, voire indispensable à l'opération traduisante?

Le professeur peut choisir de faire une présentation «théorique» du sujet. C'est alors à lui que revient la tâche d'organiser la matière et de présenter le sujet technique. Le cas échéant, il joue plus ou moins le rôle d'un spécialiste au moment où le notionnel doit l'emporter sur le linguistique.

L'enseignant peut encore laisser aux étudiants l'initiative de faire eux-mêmes une recherche sur la question. Toutefois, sans un minimum d'encadrement, cette dernière méthode est peu recommandée surtout si les étudiants ne sont pas encore rompus aux techniques de la recherche documentaire.

Quant à nous, nous avons essayé plus d'une méthode. Ainsi, devant certains groupes, nous avons déjà fait des exposés détaillés sur les sujets techniques qui allaient faire l'objet de travaux dans les semaines à venir. Cette méthode ne nous a pas semblé tout à fait satisfaisante pour la raison qu'elle n'exige pas suffisamment d'efforts de la part de l'étudiant et que ce dernier, devant le professeur qui parle, adopte plus facilement une attitude passive. En outre, cette méthode semble bien mal préparer l'étudiant à la carrière qui l'attend puisque rares sont les traducteurs professionnels qui peuvent bénéficier d'exposés détaillés sur un sujet technique de la part de spécialistes. Par ailleurs, comme il a été dit précédemment, la solution inverse qui consiste à demander aux étudiants de préparer eux-mêmes un sujet sans aucun encadrement n'est pas satisfaisante non plus : l'étudiant n'a pas encore l'habitude de constituer un dossier technique, il n'a pas non plus l'esprit formé à la pensée technique et une telle façon de procéder risque davantage de le décourager que de lui faire acquérir un début d'autonomie, ce qui devrait être l'objectif recherché.

Aux lacunes de ces deux pratiques pédagogiques opposées, nous croyons avoir trouvé une solution qui représente un juste milieu et qui s'est révélée très satisfaisante à l'expérience". Au moment d'aborder un nouveau sujet technique en classe, nous distribuons aux étudiants un dossier technique sur le sujet à l'étude, dossier que nous avons nous-même constitué. Ce dernier comporte habituellement une série de textes bilingues choisis pour leurs qualités encyclopédiques, terminologiques et didactiques. Signalons que les textes retenus sont originaux (anglais et français). En outre, le dossier peut comporter une ou plusieurs études terminologiques, dans lesquelles certains termes propres au clomaine ont fait l'objet d'une recherche approfondie. Armés d'un tel dossier, les étudiants sont en mesure de se familiariser à la fois avec l'objet technique et avec le discours technique. 
Dans un premier temps, nous demandons aux étudiants de prendre connaissance de la documentation de langue française. Il est en effet plus facile d'appréhender un nouveau domaine dans sa langue maternelle. Cette première lecture est générale et ne vise qu'à donner aux étudiants une vue d'ensemble. Dans un deuxième remps, les étudiants font une seconde lecture et sont invités à relever la terminologie qui leur semble spécifique au domaine traité et avec laquelle ils ne sont, la plupart du temps, pas familiers. Il peut s'agir d'unités terminologiques ou encore de mots ou d'expressions de la langue courante qu'ils retrouvent avec un sens différent dans le texte de spécialité. Cet exercice fait, les résultats de leur repérage terminologique sont mis en commun.

Sans condamner nécessairement la pratique qui consiste à distribuer des listes de termes aux étudiants des cours de traduction technique, nous pensons que le repérage terminologique la remplace avantageusement. En effet, comme chaque terme apparaît en contexte, l'étudiant associe plus rapidement une notion au nouveau signe et il le mémorise d'autant plus facilement.

Dans un troisième temps, les étudiants s'attachent à la compréhension et à l'organisation des notions. Par exemple, lorsque nous avons abordé le sujet de la pose de pipelines, nous leur avons demandé d'organiser, à partir de leur lecture, les différentes notions qui avaient trait à la pose elle-même et de les présenter sous la forme d'un schéma ${ }^{5}$. Ce travail effectué, un étudiant a présenté le résultat de son travail en expliquant les différentes étapes de la construction d'un pipeline, de la demande de permis pour l'emprise jusqu'à la remise en état des lieux, une fois le pipeline posé. Ce sont donc ici les étudiants qui, à partir d'un dossier technique suffisamment complet, ont dû faire l'effort de compréhension nécessaire pour organiser eux-mêmes les notions et faire correspondre un signe.

Quand ils ont suffisamment maîtrisé le contenu de la documentation française, les étudiants sont prêts à s'attaquer aux documents parallèles de langue anglaise. La démarche suivie est sensiblement la même que pour les textes français: première lecture pour avoir une vue d'ensemble des textes, seconde lecture où la terminologie pertinente sera repérée. Toutefois, comme les textes français et anglais peuvent varier quant à leur contenu informatif, il est possible que la lecture des textes anglais apporte de nouveaux renseignements. On prendra alors soin de compléter le schéma déjà établi en ajoutant les données importantes qui n'auraient pas figuré dans les textes français. Enfin, après avoir vérifié avec les étudiants les correspondances entre les termes anglais et français, le repérage des termes anglais pourra aussi être reporté sur le schéma. De cette façon, les étudiants disposeront d'un lexique de base qu'ils auront eux-mêmes constitué à partir de la langue actualisée et qui leur sera utile au moment d'entreprendre la traduction de textes.

Sur le plan de l'apprentissage, les étapes que nous venons de décrire sont préalables à tout acte de traduction. À ce titre, chacune de ces étapes doit constituer un moyen d'atteindre l'objectif fixé, cet objectif étant l'acquisition d'un contenu technique et d'une compétence linguistique, dont on devra vérifier les résultats effectifs ${ }^{6}$.

Voyons maintenant les résultats d'un extrait du texte didactique sur la pose de pipelines que nous avons donné à traduire à nos étudiants.

Le texte à l'étude a trait à la pose de pipelines, plus précisément d'oléoducs. Il est extrait d'un manuel didactique intitulé Fundamentals of Petroleum et publié par The University of Texas at Austin. Il s'adresse particulièrement aux étudiants en génie et à tout spécialiste œuvrant dans le domaine du transport de l'énergie. 


\title{
PIPELINE CONSTRUCTION ${ }^{7}$
}

\section{LAYING PIPE ON LAND}

Cleaning, aligning, welding, and coating pipe

\begin{abstract}
Before the pipe is welded in place, the ends must be absolutely clean of all dirt, scale, and coating. Sometimes the beveled edges of the joint must be recut before the two pipes ends are aligned. To accomplish alignment, clamps are used outside on small-diameter pipe and inside on larger-diameter pipe; the side-boom tractor and winches lower the pipe into place and hold it while skids are put under it and the initial welds are made. The welds are then inspected visually and by $\mathrm{X}$ rays before the pipe is coated and wrapped. A machine cleans the pipeline with rotating brushes, and a layer of fast-drying enamel is applied to aid in binding the wrapping at the joints. Then a protective coating of coal tar or asphalt is applied ; a layer of glass fiber follows, coated at temperatures of 475 to 575 degrees Fahrenheit, and a final coating of asbestos felt or tar paper is wrapped around the whole thing to protect the coating from damage by skids, slings, or rocks during lowering of the line and backfilling operations.
\end{abstract}

\section{Lowering in}

In some conditions pipe is lowered into the ditch in conjunction with the coating and wrapping operation. This method eliminates the two steps of lowering the pipe to skids and picking it up later for final placement.

Crossing preexisting pipelines requires going under the line already in place. In some situations six or more side-boom tractors must be used in the operation. Creek crossings and steep terrain also require the skillful maneuvering of side-boom operators as they move long sections of pipe into position.

\section{Tying in}

Highway, railroad, and river crossings leave openings in the line that are close by the tie-in crew, a miniature spread that joins the segments into one continuous line. At tie-ins, extra pipe is cut off and bevels made, welds are made, and protective coatings are put on, until the entire line is complete.

On sait que pour traduire un texte technique, le traducteur doit non seulement maîtriser deux langues, mais avoir aussi une connaissance du sujet traité. Deux types de connaissances entrent donc en ligne de compte: connaissances linguistiques et connaissances thématiques. Avant de traduire le texte sur les pipelines, les étudiants avaient acquis un minimum de connaissances thématiques sur le sujet. Les résultats furent probants et, dans l'ensemble, aucune erreur n'était attribuable à la mauvaise compréhension résultant d'une méconnaissance du sujet. Il faut sans doute préciser que, le texte à traduire étant de nature descriptive et assez semblable de par son contenu aux textes parallèles du dossier technique, l'étudiant qui possédait bien son dossier ne devait normalement pas avoir de difficultés de compréhension.

Par contre, il est intéressant de noter que la plupart des erreurs relevées avaient trait à l'imprécision lexicale. La précision, l'une des quatre qualités que nous prêtions au texte de spécialité, est l'élément qui a fait le plus défaut dans les traductions des étudiants.

Examinons maintenant les erreurs lexicales qui, dans l'ensemble, sont le résultat d'un transcodage inconsidéré et de l'utilisation peu judicieuse des dictionnaires. 
Tout d'abord, dans l'un des sous-titres du texte, le terme welding a donné lieu à deux traductions, soit «soudage» et «soudure». Comme il s'agissait ici de l'opération elle-même, et non du résultat, seul le terme «soudage» devait être utilisé, le terme «soudure» s'appliquant au résultat du soudage.

En outre, la phrase «Before the pipe is welded in place, the ends must be absolutely clean of all dirt, scale and coating» a donné lieu chez certains étudiants à une imprécision lexicale qui a entraîné un faux sens. Le terme coating a notamment été rendu par «enrobage», «couche d'enrobage», «revêtement» et «traces d'enrobage». Pour qui connait un peu le domaine et a bien pris connaissance du texte, il est évident qu'il ne peut s'agir ici d'enrobage ni de revêtement. En effet, le terme coating apparaît en début de texte, à un moment où l'on n'en est qu'aux étapes préliminaires à l'enrobage, et plus précisément au nettoyage des tubes.

Comme les étudiants avaient appris que le terme coating revêt une acception particulière lorsqu'il est utilisé dans les étapes de pose de pipelines, et qu'il a comme correspondant le terme «enrobage», ils ont, sans même se poser la question, utilisé ce correspondant qui aurait pu être juste dans un autre contexte. Toutefois, le terme coating était utilisé ici dans son sens technique général, à savoir toute couche d'un produit qu'on applique, et avait alors pour correspondant le terme «enduit». La phrase anglaise précisait simplement qu'avant de procéder au soudage des tubes, il faut s'assurer que les extrémités ne présentent aucune impureté, écaillure, ou enduit.

Il est certain qu'un manque de réflexion est ici à l'origine de l'erreur. En utilisant les termes «enrobage» et «revêtement» pour traduire coating, les étudiants auraient dû se rendre compte, ne serait-ce qu'à la lecture finale de leur texte, qu'il n'était logiquement pas possible de faire disparaître l'enrobage sans même l'avoir appliqué. C'est donc la dernière signification acquise du terme anglais et son correspondant français qui ont surgi à leur esprit et qu'ils ont vite fait d'utiliser.

Paradoxalement, serait-ce dans un souci de précision que certains étudiants ont commis une erreur de surtraduction? Ainsi, dans la phrase «The welds are then inspected visually and by $\mathrm{X}$ rays before the pipe is coated and wrapped», quelques étudiants ont vu dans coated and wrapped deux étapes différentes et ont donc traduit cette unité par «avant que les tubes soient revêtus et enroulés». Si en anglais deux mots sont nécessaires pour désigner l'étape qui consiste à mettre en place, sur la conduite, un revêtement destiné à la protéger contre les agents corrosifs, un seul mot en français correspond à cette signification. Il s'agit du terme «enrobage» ou de son synonyme «revêtement». Enfin, un étudiant a traduit cette unité par «avant l'enrobage et l'emballage des tubes». Non content de faire une surtraduction, cet étudiant n'a pas identifié la signification supplémentaire propre à ce mot dans la langue de spécialité.

Enfin, le terme line, qui figurait à quatre reprises dans le texte, a été mal rendu par la moitié des étudiants. Une fois de plus, c'est l'acception la plus courante qui a été retenue et l'on a traduit ce terme par «ligne». Bien que dans deux cas cette solution ait pu sembler plausible aux étudiants a priori, dans les deux autres, le contexte montrait clairement qu'il ne pouvait s'agir d'une «ligne»:

[...] a final coating of asbestos felt or tar paper is wrapped around the whole thing to protect the coating from damage by skids, or rocks during lowering of the line and backfilling of operations.

At tie-ins, extra pipe is cut off and bevels made, welds are made, and protective coatings are put on, until the entire line is complete.

Dans les quatre contextes où il apparaissait, le terme line désignait «a system of pipes for distribution» et il aurait donc dû se traduire par «canalisation» ou encore par 
«conduite». Enfin, on aurait aussi pu rendre le terme line par «pipeline», les contextes le permettant.

La correction de ce texte a été suivie d'une séance de discussion. Cette séance est aussi importante pour les étudiants que pour l'enseignant. Si les étudiants y trouvent l'occasion de comprendre leurs erreurs, l'enseignant, quant à lui, pour peu qu'il consente à aller plus à fond et à interroger les étudiants sur les raisons qui les ont motivés à faire un choix plutôt qu'un autre dans leur traduction, en apprend beaucoup sur la source des erreurs commises. Ces séances peuvent donc lui être très utiles du point de vue pédagogique. En tenant compte des lacunes observées, il pourra mettre au point des exercices particuliers pour combler de telles lacunes. Par exemple, ce sont les mauvaises traductions répétées des termes spécialisés empruntés à la langue courante, comme line, head, shoulder, etc., qui nous ont incitée à mettre au point un exercice qui vise à faire travailler les étudiants sur l'interférence entre la langue générale et la langue de spécialité.

Par ailleurs, l'analyse des erreurs révèle que c'est avec l'introduction des textes didactiques que se pose de façon plus évidente le problème de la terminologie et, par conséquent, celui de l'imprécision lexicale. Deux catégories de termes posent des difficultés aux étudiants: les termes exclusifs à la langue de spécialité et les termes qui ont une double acception, l'une dans la langue générale, connue des étudiants, l'autre dans la langue de spécialité, inconnue de ceux-ci.

Dans un premier temps, la recherche par les étudiants de correspondants de termes exclusifs à la langue de spécialité donne de mauvais résultats pour deux raisons. D'abord, parce que les étudiants retiennent la plupart du temps le premier terme de la liste de correspondants fournis par le dictionnaire, sans égard au domaine d'emploi du terme recherché. Ensuite, parce que les étudiants, en raison de leur manque d'expérience, se servent parfois de dictionnaires ou d'ouvrages dont la fiabilité, sur le plan de la terminologie, laisse à désirer. Pédagogiquement, on peut viser à combler de telles lacunes en intégrant à l'enseignement un ou plusieurs exposés sur les différents types de dictionnaires et la façon de les consulter, assortis d'un exercice pratique sur la recherche de correspondants. Enfin, il faut aussi fournir aux étudiants les moyens pratiques pour évaluer la documentation dont ils se servent.

Dans un deuxième temps, les termes qui ont une double acception, dont l'une dans la langue générale, qui est connue des étudiants, et l'autre dans la langue de spécialité, qui leur est inconnue, ne donnent généralement lieu à aucune recherche de leur part. En effet, connaissant l'acception de ces termes dans la langue générale, les étudiants ne se doutent pas que ces termes peuvent avoir une acception différente dans la langue de spécialité. Sur le plan de la pédagogie, l'enseignant doit mettre les étudiants en garde contre les termes qui ont une double acception de façon à créer chez eux un automatisme de doute du même genre que celui qu'ils ont acquis pour les anglicismes. Enfin, des exercices sur la traduction de termes empruntés à la langue générale les aideront, premièrement, à prendre conscience de l'occurrence relativement fréquente de ces termes dans les textes techniques et, deuxièmement, à introduire dans leurs traductions des correspondants justes.

\section{NOTES}

1. Amy Mereson (1985): «Asbestos: The Problem Grows» dans Science Digest, January, p. 32.

2. Si le terme asbestos n'a pas été mentionné, c'est tout simplement parce que les étudiants croyaient le connaître, le Québec étant un grand producteur d'amiante et une de ses villes portant même le nom d'Asbestos. Il en va de même pour asbestosis puisque les médias canadiens ont fréquemment fait état de l'amiantose et de ses ravages chez les travailleurs de l'amiante.

3. C'est nous qui soulignons. 
4. Il faut rappeler ici que les considérations pédagogiques qui font l'objet du présent article s'appliquent à l'enseignement d'un cours de traduction technique de premier niveau et que, par conséquent, elles ne sont pas nécessairement toutes valables pour les cours de niveaux plus avancés.

5. Comme la plupart des textes qui composaient le dossier technique sur la pose de pipelines étaient descriptifs, l'utilisation du schéma se prêtait bien à cet exercice.

6. L'acquisition d'un contenu technique et d'une compétence linguistique doit faire partie des objectifs d'apprentissage au même titre que l'acquisition d'une méthode de traduction technique. L'enseignant doit donc mesurer cette acquisition au moyen de contrôles des connaissances sur le sujet technique à l'étude ainsi que sur la terminologie et la phraséologie qui s'y rapportent. Comme le dit J. Delisle $(1988: 213)$ au sujet du contrôle des connaissances dans l'enseignement de la traduction économique : «Puisque traduire est un savoir-faire (interpréter et réexprimer) reposant sur un double savoir (linguistique et encyclopédique), il importe, lors des tests ou des examens, de faire porter l'évaluation à la fois sur les connaissances et sur les aptitudes acquises. C'est pourquoi tout ce qui a été vu durant le séminaire est matière à examen : le vocabulaire, la matière ayant fait l'objet des exposés et des exercices (phraséologiques, grammaticaux, stylistiques, terminologiques), les corrections des traductions annotées [...].»

7. Petroleum Extension Service (1979): Fundamentals of Petroleum, Austin, University of Texas, p. 201.

\section{RÉFÉRENCES}

CARY, Edmond (1985): Comment faut-il traduire?, Introduction, bibliographie et index de Michel Ballard, Lille, Presses universitaires de Lille, $95 \mathrm{p}$.

CORMIER, Monique C. (1990) : «Proposition d'une typologie pour l'enseignement de la traduction technique», Marianne Lederer (dir.), Les Études traductologiques, Paris, Minard-Lettres Modernes.

CORMIER, Monique C. et Pierre MÉNASSA (1987): «Le Québec produit-il de l'amiante ou de l'asbeste ?», Meta, 32-4, pp. 450-455.

DELISLE, Jean (1980): L'analyse du discours comme mêthode de traduction, Ottawa, Éditions de l'Université d'Ottawa, $282 \mathrm{p}$.

DELISLE, Jean (1988): «L'initiation à la traduction économique», Meta, 33-2, pp. 204-215.

LEDERER, Marianne (1981): La traduction simultanée, Paris, Minard-Lettres Modernes, $454 \mathrm{p}$.

SELESKOVITCH, Danica (1975) : Langage, langues et mémoire, Paris, Minard-Lettres Modernes, 272 p. 